\title{
The Prevelance of Insulin Resistance in Turkish Population
}

Ahmet Kaya1, Elif Turan2, Mehmet Uyar3, Fahri Bayram4, Yasar Turan5

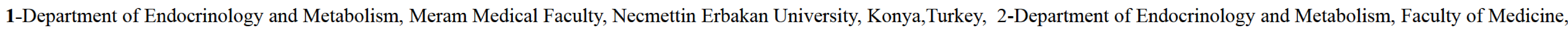
Bozok University, Yozgat, Turkey , 3-Department of Public Health, Meram Medical Faculty, Necmettin Erbakan University, Konya,Turkey , 4-Department of Endocrinology and Metabolism,

Faculty of Medicine, Erciyes University, Kayseri, Turkey, 5-Department of Cardiology, Faculty of Medicine, Bozok University, Yozgat, Turkey

\section{OBJECTIVES}

The insulin resistance (IR) is a pathogenic factor for type 2 DM $(1,2)$ IR form an insensitivity of the peripheral tissues (for example adipose tissue, liver, muscle) to the impact on insulin. Insulin resistance and related situations are very common and $30-40 \%$ of subjects who live in affluent countries are affected. Also IR is a common finding in developing countries .

The homeostasis model assessment of insulin resistance (HOMA-IR) is an non invasive and effective method to evaluate insulin sensitivity from the glucose and level of serum insulin measured as fasting conditions. HOMA-IR is recieved as the standard method to measure insulin resistance in epidemiologic studies

We aimed to investigate the homeostasis model assessment of insulin resistance (HOMA-IR) and relation of HOMA-IR with age, sex and body mass index (BMI) and the prevelance of diabetes in Turkish population of different region.

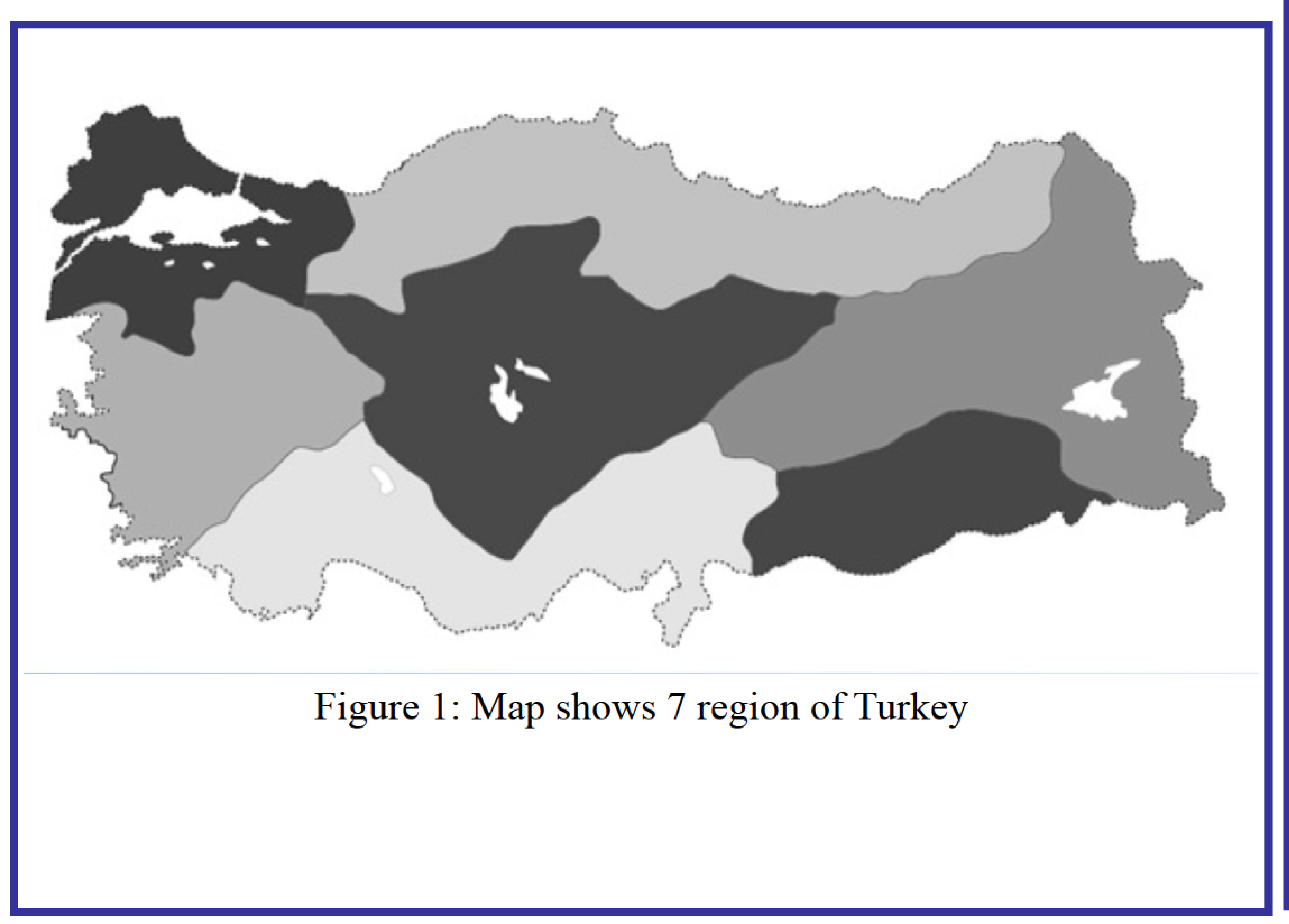

\section{CONCLUSIONS}

This study reported that there was a significant prevalence of diabetes, IFG and IR in 7 regions of Turkey and also we reported the distribution of HOMA-IR, an a accepted measure of insulin resistance in a population-based sample of Turkish people, results showed that HOMA-IR is significantly higher in obese compared with normal-weight participants.

The high prevelance of IR and DM in Turkey is one of the important public health problem. There is an urgent necessity to institute more aggressive nation wide public health measures and screening programs about obesity.

\section{METHODS}

The insulin resistance (IR) is a pathogenic factor for type 2 DM $(1,2)$. IR form an insensitivity of the peripheral tissues (for example adipose tissue, liver, muscle ) to the impact on insulin. Insulin resistance and related situations are very common and $30-40 \%$ of subjects who live in affluent countries are affected. Also IR is a common finding in developing countries .

The homeostasis model assessment of insulin resistance (HOMA-IR) is an non invasive and effective method to evaluate insulin sensitivity from the glucose and level of serum insulin measured as fasting conditions. HOMA-IR is recieved as the standard method to measure insulin resistance in epidemiologic studies

We aimed to investigate the homeostasis model assessment of insulin resistance (HOMAIR) and relation of HOMA-IR with age, sex and body mass index (BMI) and the prevelance of diabetes in Turkish population of different region.

The insulin resistance (IR) is a pathogenic factor for type 2 DM $(1,2)$. IR form an insensitivity of the peripheral tissues (for example adipose tissue, liver, muscle ) to the impact on insulin. Insulin resistance and related situations are very common and $30-40 \%$ of subjects who live in affluent countries are affected. Also IR is a common finding in developing countries

The homeostasis model assessment of insulin resistance (HOMA-IR) is an non invasive and effective method to evaluate insulin sensitivity from the glucose and level of serum insulin measured as fasting conditions. HOMA-IR is recieved as the standard method to measure insulin resistance in epidemiologic studies. We aimed to investigate the homeostasis model assessment of insulin resistance (HOMA-IR) and relation of HOMA-IR with age, sex and body mass index (BMI) and the prevelance of diabetes in Turkish population of different region.

\section{References}

\section{References}

1. Bonora E, Kiechl S, Willeit J, Oberhollenzer F, Egger G, Meigs JB, Bonadonna RC, Muggeo M. ( 2004) Population-based incidence rates and risk factors for type 2 diabetes in white individuals: the Bruneck Study. Diabetes 53:1782-1789.

2. Weyer C, Bogardus C, Mott DM, Pratley RE (1999) The natural history of insulin secretory dysfunction and insulin resistance in the pathogenesis of type 2 diabetes mellitus. J Clin Invest 104:787794 